\title{
RISCO DE INFECÇÃO TUBERCULOSA EM MUNICÍPIO DO INTERIOR DO ESTADO DE SÃO PAULO E SUAS APLICAÇŌES *
}

Rlffino NetTo. A. \& Arantes, G. R. - Risco de infeccão tuberculosa em municipio do interior do Estado de são Paulo e suas aplicacóes. Rev. Saúde públ., S. Panlo, 10:143-9, 1976.

RESUMO: Foi proposto estimar o risco de infeccáo tuberculosa na pojulação de Ribeirão Preto, SP (Brasul) a partir de estudo transiersal, assim como a incidencia anual de casos bachliferos nessa comuniade. As estimativas foram efetuadas com dados de 35.680 pessoas residentes no municipio e ane foram cadastradas na Unidade Sanitaria local. Foi estimado o risco de infecção para diferentes grupos etários e para a população geral, bem como analisadas suas vamaçóes e implicaçós.

Unitermos: Infecsão tuberculosa risco. Prevaléncia e incidencia. São Palulo, Brasit.

\section{N T P ODLÇĀO}

O programa antituberculose executado pelas Lnidades Sanitárias do Estado de São Paulo é constituído lasicamente por três alividades: descoherta de casos, tratamento e proteção aos susceptiveis.

() efeito gerado pelo programa. isto é. a impacto prorocado na magnitude do problema. será consequiência da soma das efirácias alcançadas em cada uma das atividades que o compóem.

Sabemos que. a partir da prevalência de reatores à prova tulierculinica lem diferentes grupos etários) é posível calcular o risco de infecção tuberculosa nes- sa população \&. Stỵlo \& Sutherland $119711^{\circ}$ propõem que a partir do conhecimento do risco de infecção seria possível estimar a incidência anual de meningite tuberculosa. assim como incidencia anual de morbidade una ausência de programas de controle para a doença).

Analisando os dados referentes a pessoas que demandam ao Centro de Saúde de Rilierão Preto. SP. Brasil de diferenles grupos etários). propusemo-nos no presente trabalho a múltiplos objetiros:

1. estimar o risco de infecção tuberculosa nessa população;

\footnotetext{
* Apresentado no XVIr Congresso Nacionai de Tuberculose e Doencas Respiratórias -Brasilla, DF, 1975.

** Da Faculdade de Medicina de Ribejrão Preto da LSP - Ribeirão Preto, SP — Brasil

*** Da 6.a Divisão Regional de Saúde da Secretaria da Saúde do Estado de São Paulo Rua Minas, 895 - Ribeirão Preto, SP — Brasil.
} 
RUFFINO NETTO, A. \& ARANTES, G. R. - Risco de infecção tuberculosa em municipio do Interior do Estado de São Paulo e suas aplicações. Rev. Saúde públ., S. Paulo, $10: 143-9,1976$.

2. estimar a incidência de tuberculose a partir do risco de infecção;

3. cotejar a incidência esperada e a incidência observada (detectada pelo Centro de Saúde ou notificada ao mesmo) para estudar possivel aplicabilidade deste método para avaliar a eficácia da atividade "descoberta de casos" executada no programa de controle da tuberculose.

\section{MATERIAL E METODOS}

O município de Ribeirão Preto, cuja população era de 235.231 habitantes em 1/9/73, possui um Centro de Saúde Polivalente, de cujo programa constam atividades para controle da tuberculose. Para isso conta com uma Clínica Tisiológica atendida por médicos especialistas, abreugrafia, radiologia, teste tuberculínico padronizado, exames bacteriológicos (realizados no laboratório do Instituto Adolfo Lutz), enfermagem e visitação domiciliar. 0 descobrimento de casos é feito, passivamente, entre as pessoas que afluem à Unidade espontaneamente ou para obter certificados de saúde; o serviço de visitação domiciliar é insatisfatório, limitando-se ao chamamento de doentes faltosos e comunicantes cujo comparecimento é muito inferior ao previsto ${ }^{1}$. Não há trabalho de educação junto à comunidade visando um maior comparecimento de pessoas com sintomatologia respiratória.

Os casos diagnosticados por outras fontes de atenção médica foram conhecidos através de relatórios que os laboratórios de análises, por força de lei, enviam mensalmente ao Centro de Saúde, contendo o nome e endereço das pessoas que apresentaram exames positivos para doenças de notificação compulsôria.

A prevalência de infectados (reatores fortes) por grupos etários foi obtida pela aplicação do teste tuberculínico padronizado (com 2 T.U. de PPD rt 23) a todas as pessoas residentes no município, com mais de 3 meses de idade, que se matricularam no Centro de Saúde durante 12 meses consecutivos (maio de 1973 a abril de 1974).

O coeficiente ou risco anual de infecção foi calculado pela fórmula:

$$
r=\frac{1}{t} \log \text { natural } \frac{N o}{N}
$$

derivada da expressão:

$$
\mathrm{N}=\mathrm{No}_{\mathrm{o}}-\mathrm{rt}
$$

sugerida por Ruffino Netto ${ }^{4}$ (1975), para estudos longitudinais, onde:

$$
\begin{aligned}
\text { No }= & \text { número de pessoas virgens de } \\
& \text { infecção no início da contagem } \\
& \text { do tempo (ou seja para tempo } \\
& t=0) ; \\
\mathrm{N}= & \text { número de pessoas que ainda } \\
& \begin{array}{l}
\text { permanecem virgens de infecção } \\
\text { (entre os } N o \text { iniciais) decorrido }
\end{array} \\
& \text { certo tempo } t ; \\
\mathrm{t}= & \text { tempo decorrido em anos; } \\
\mathrm{e}= & \text { base do logarítimo neperiano ou } \\
& \text { natural; } \\
\mathrm{r}= & \text { risco anual de infecção. }
\end{aligned}
$$

Embora a fórmula tenha sido deduzida para estudos longitudinais, poderá ser utilizada em estudos transversais se chamarmos $N o$ e $N$ as percentagens de não reatores em dois grupos etários consecutivos; $t$ é o intervalo entre os pontos médios dos dois grupos etários e $r \quad 0$ risco de infecção. (Observar que a fórmula $N=N_{o} e^{-r t}$ é equivalente àquela sugerida por Narain e col. (1966) ${ }^{3}$, $q^{\prime}=q^{e-r n}$, embora tenha sido deduzida por caminho diferente). 
RUFFINO NETTO, A. \& ARANTES. G. R. - Risco de infecção tuberculosa em município do interior do Estado de São Paulo e suas aplicações. Rev. Saúde públ., S. Paulo, $10: 143-9,1976$.

\section{RESULTADOS E DISCUSSÃO}

A demanda estudada embora não sendo uma amostra casual da população, provavelmente expresse a sensibilidade tuberculínica da mesma, pelas razōes apresentadas em outra publicação?

Os resultados da aplicação do teste tuberculínico, lido em 35.680 pessoas em função dos grupos etários, são apresentados na Tabela 1.

Assim, supondo que ao nascimento $100 \%$ das pessoas sejam não reatores e que no grupo etário de 0 até 15 anos a prevalência de negativos seja de $95.1 \%$ podemos tomar o tempo como o intervalo entre 0 e 7.5 (ponto médio entre 0 e 15) e calcular o risco de infecção:

$$
\begin{gathered}
r=\frac{1}{t} \log \text { natural } \frac{N_{0}}{N}= \\
\frac{1}{7,5} \log \text { natural } \frac{100}{95,1}=0.6699 \digamma_{c}
\end{gathered}
$$

ou seja, o risco de infecção no grupo etário de 0 a 15 anos foi de $0,6699 \%$.

Outros exemplos:

a) o risco de infecção no grupo etário de 15 a 20 anos será:

$$
\begin{aligned}
& r=\frac{1}{10} \log \text { natural } \frac{95.1}{86,9}= \\
& =0,9017 \%
\end{aligned}
$$

onde o tempo é igual a 10 , isto é, o intervalo entre 7.5 (ponto médio de 0 a 15) e 17.5 (ponto médio de 15 a 20 ).

b) o risco de infecção no grupo etário de 20 a 25 anos será:

$$
\begin{aligned}
& \mathbf{r}=\frac{1}{5} \log \text { natural } \frac{86.9}{79.2}= \\
& =1,8556 \%
\end{aligned}
$$

\begin{tabular}{|c|c|c|c|c|c|}
\hline \multirow{2}{*}{ Grupo etário } & \multicolumn{2}{|c|}{ Não reatores * } & \multicolumn{2}{|c|}{ Reatores } & \multirow{2}{*}{$\begin{array}{c}\text { Total de } \\
\text { cadastrados }\end{array}$} \\
\hline & $\mathrm{N} \cdot{ }^{\circ}$ & $\%_{0}$ & N.o & $\%$ & \\
\hline $01-15$ & 4.563 & 95,1 & 235 & 4,9 & 4.798 \\
\hline $15-20$ & 8.362 & 86,9 & 1.258 & 13,1 & 9.620 \\
\hline 20 - 25 & 5.309 & 79,2 & 1.396 & 20,8 & 6.705 \\
\hline $25 !-30$ & 3.084 & 74,1 & 1.080 & 25,9 & 4.164 \\
\hline $30-35$ & 2.027 & 67.9 & 958 & 32.1 & 2.985 \\
\hline $35-40$ & 1.440 & 63,2 & 837 & 36,8 & 2.277 \\
\hline $40-45$ & 1.078 & 60,4 & 706 & 39.6 & 1.784 \\
\hline $45-50$ & 712 & 54.9 & 586 & 45,1 & 1.298 \\
\hline $50 \mid-55$ & 478 & 54,3 & 402 & 45,7 & 880 \\
\hline $55 \mid-60$ & 276 & 51,6 & 259 & 48,4 & 535 \\
\hline $60 \mid-65$ & 166 & $\mathbf{5 0}, 2$ & 165 & 49.8 & 331 \\
\hline $65 !-70$ & 88 & 52,4 & 80 & 47,6 & 168 \\
\hline $70 \mathrm{e}+$ & 80 & 59,3 & 55 & 40.7 & 135 \\
\hline Total & 27.663 & 77.5 & 8.017 & 22.5 & 35.680 \\
\hline
\end{tabular}

T A B E L A 1

Distribuição dos reatores à prova tuberculínica (tuberculina PPD $r t \quad 23-2 \quad$ T.U.) segundo grupo etário - Centro de Saúde de Ribeirão Preto, SP — 1973

* Com diâmetros de induraşāo inferiores a $10 \mathrm{~mm}$. 
RLFFINO NETTO, A \& ARANTES. G. R. - Risco de infecsão tuberculosa em municipio do interior do Estado de São Paulo e suas aplicacóes. Rev. Saúde públ., S Paulo, 10:143-9, 1976.

Ohserve-se que o tempo igual a 5 é o intervalo entre 17.5 (ponto médio de 15 a 201 e 22.5 (ponto médio de 20 a 25 ).

Ta Tabela 2 são apresentados os riscos anuais de infecção para os diferentes grupos etários. Cerca de $91 \Upsilon^{\prime} c$ da população cadastrada se encontra abaixo da idade de 45 anos iTabela 1) ou $94 \%$ ahaixo da idade de 50 anos. Realmente. a partir dos 50 anos. o número dos cadastrados rai caindo progressivamente. Por estas razóes. e acrescido o fato de nos preocuparmos mais com o risco de infecção ahaixo dos 50 anos. iremos nos deter mais no intervalo de 0 a 50 anos, onde ohservamos que o " $r$ " varia de 0.6699 a $1.9095^{\prime} r$ r. com un valor mediano de $1.3820 r^{\prime c}$. Ohservamos que o " $r$ " aumenta com idade até o máximo dos 25 anos $11.85560^{\circ}, 1$ a seguir decrescendo até $0.906: 3$.

Em estudo longitudinal levado a efeito entre escolares I de $1+t$ escolas primárias? no grupo tário de 7 a 12 anos. na (iidade de Riheirāo Preto. Ruffino Vetto e col." 1975 ) encontraram o risco anual de infecção tuherculosa de 1.3290 'c. valor ese bem proximo aos valores encontrados no presente trahalho. e especificamente ao valor mediano $(1.382 \%)$ entre os riscos assinalados para os rários grupos etários compreendidos entre 0 a 50 anos.

A partir do conhecimento do risco de inferção nos diferentes grupos etários lcalculado através do estudo transerersali. procuramos calcular qual seria o risco de infecção na população geral ou em outros diferentes extratos etários.

Lembrando a expressāo (2)

$$
x=\lambda e^{-11} \text {. }
$$

o número de parientes infectado- 1J! em verto tempo l selá:

$$
1=10-V_{0}-1 t
$$

ou

$$
I=\gamma_{0}\left[1-e^{-r t}\right]
$$

Estimamos a população total nos diferentes grupos etários da cidade de Riheirão Preto (Tabela 3), estimando em seguida o número de pessoas não infectadas tho dentro de cada subgrupo). Conhecendo-se para cada extrato ou grupo etário o valor de $\mathrm{No}$ e de " $r$ ", podemos aplicar a fórmula it) e estimar o número esperado de infectados (I) em 1 ano.

\section{TABELA 2}

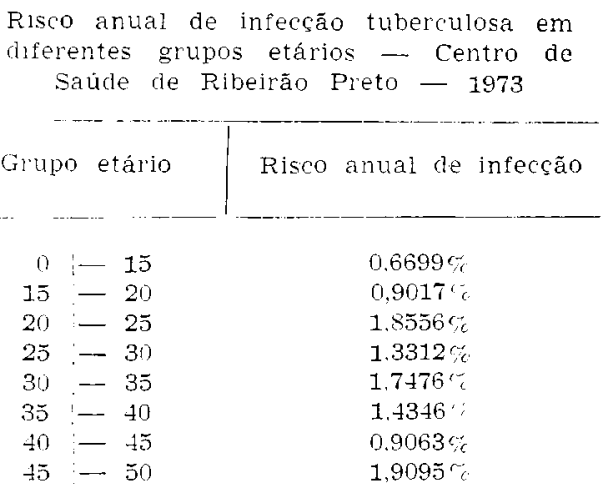

Ia Tahela 3 apresentamos extas estimativas.

Efetuando o raciocínio inverso, ișto é, dados agora os valores de $l$. No e $t$ podemos calcular o $r$ tpara qualquer composição etária). on seja:

$$
\begin{aligned}
& 1=X_{0}\left[1-e^{-1 t}\right]=X_{0}-\mathrm{Noe}_{0}[t \\
& \mathrm{Xo}^{-H}=\mathrm{Yo}_{\mathrm{O}}-\mathrm{I} \\
& r=\frac{1}{t} \quad \log \text { natural } \quad \begin{array}{c}
\text { No } \\
\text { No }-I
\end{array}
\end{aligned}
$$

Exemplo: o risco de infeç̧ão geral para a população de 0 a 65 anos será, no decorrer de um ano: 
RUFFINO NETTO, A. \& ARANTES, G. R. - Risco de infecção tuberculosa em municipio do interior do Estado de São Paulo e suas aplicações. Rev. Saúde públ., S. Paulo, 10:143-9, 1976.

T A B E L A 3

Estimativas de algumas variáveis concernentes ao município de Ribeirão Preto, em função do grupo etário - 1973

\begin{tabular}{|c|c|c|c|c|c|}
\hline $\begin{array}{l}\text { Grupo } \\
\text { etário }\end{array}$ & $\begin{array}{l}\text { População } \\
\text { estimada }\end{array}$ & $\begin{array}{l}\% \text { de nāo } \\
\text { infectados }\end{array}$ & $\begin{array}{l}\text { N. estimado } \\
\text { de não } \\
\text { infectados } \\
\text { (No) }\end{array}$ & $\begin{array}{l}\text { Risco de } \\
\text { infecção } \\
\text { em \% } \\
\text { (r) }\end{array}$ & $\begin{array}{l}\text { N.o estimado } \\
\text { de infectados } \\
\text { por ano } \\
\text { (I) }\end{array}$ \\
\hline $01-15$ & 82.364 & 95,1 & 78.328 & 0,6699 & 523 \\
\hline $15 j-20$ & 26.682 & 86,9 & 23.186 & 0,9017 & 209 \\
\hline $20 i-25$ & 22.968 & 79,2 & 18.190 & 1,8556 & 335 \\
\hline $25 \mid-30$ & 18.025 & 74,1 & 13.356 & 1,3312 & 177 \\
\hline $301-35$ & 15.896 & 67,9 & 10.793 & 1,7476 & 187 \\
\hline $35 j-40$ & 14.878 & 63,2 & 9.402 & 1,4346 & 134 \\
\hline $401-45$ & 13.672 & 60,4 & 8.257 & 0,9063 & 75 \\
\hline $451-50$ & 10.574 & 54,9 & 5.805 & 1,9095 & 110 \\
\hline $50 i-55$ & 8.965 & 54,3 & 4.867 & 0,2198 & 11 \\
\hline $55 \mid-60$ & 7.192 & 51,6 & 3.711 & 1,0201 & 38 \\
\hline 60 i- 65 & 5.842 & 50,2 & 2.932 & 0,5501 & 17 \\
\hline Total & 227.058 & - & 178.827 & 一 & 1.816 \\
\hline
\end{tabular}

$r=\frac{1}{1} \log$ natural

$$
\begin{gathered}
\frac{178.827}{178.827-1816}=0,01021 \\
\text { ou } \\
r=1,021 \%
\end{gathered}
$$

$\mathrm{Na}$ Tabela 4 apresentamos o risco de infecção $(\mathrm{em} \%)$ estimado para diferentes extratos etários da população estudada. Chama a atenção nesta tabela que o " $r$ " assume valores crescentes em função de idade. Por outro lado, quando tomamos os estratos maiores de 20 anos, encontramos os grandes valores de " $r$ ". Assim, de 20 - 50, foi encontrado 1,4890 .

$\mathrm{Na}$ Tabela 2 também se observa que ocorre um grande salto quando se passa do grupo etário $15 \mid-20(r=0,9017 \%)$ para o grupo $20 \mid-25$ ( $r=1,8556 \%)$.

Parece que as coortes de $0-20$ ou $0 \mid-25$ anos apresentaram probabilida- des de se infectarem durante estes seus anos de vida, bem menores que as coortes de idades superiores a 20 ou 25 anos. Se levarmos em consideração que a coorte de 0 a 25 (em 1973) constituem as pessoas nascidas a partir de 1950 ou 1955 , concluiremos que seguramente as fontes de infecção no município devem ter sido sensivelmente reduzidas a partir desta época. É possível que o fato esteja relacionado ao aparecimento e aplicação em maior escala dos quimioterápicos específicos.

Conhecido o risco de infecção, o nosso segundo objetivo seria estimar a incidência anual de casos bacilíferos em pessoas residentes no municipio. Segundo Styblo \& Sutherland ${ }^{6}$ (1974), para países de alta prevalência de tuberculose a incidência anual de casos bacilíferos à baciloscopia (todas as idades e por cem mil habitantes) é 60 vezes maior que o risco de infecção anual $(r \%)$. Se assumirmos esta relação como válida, para $r=1,0210 \%$ (risco geral da população de 0 a 65 anos em Ribeirão Preto) seria esperada, portanto, uma incidência anual 
RLFFINO NETTO, A. \& ARANTES, G. R. - Risco de infeção tuberculosa em municipio do interior do Estado de São Paulo e suas aplicações. Rev. Saúle públ., S. Paulo, 10:143-9, 1976 .

de $61.26 / 100.000$. ou seja. 140 casos nessa população.

T A B E A 4

Estimativa do risco de infeccão em diferentes grupos etários de Ribeirão Preto - 1973

\begin{tabular}{|c|c|c|}
\hline \multicolumn{2}{|c|}{ Grupo etário } & $\mathrm{r}(\%)$ \\
\hline 0 & $1-20$ & 0,6980 \\
\hline 0 & $1-25$ & 0.7970 \\
\hline 0 & $1-50$ & 0,9950 \\
\hline 0 & $1-65$ & 1,0210 \\
\hline 20 & $1-\quad 50$ & 1.4890 \\
\hline 25 & -50 & 1,3900 \\
\hline
\end{tabular}

No periodo de maio de 1973 a abril de 1974, a L'nidade Sanitária de Ribeirão Preto descobriu 57 casos de tuberculose pulmonar confirmada hacteriologicamente em pessoas residentes no município (das quais $37 \quad 165 \%$, positivas à baciloscopia). Outros 8 casos foram diagnosticados fora da Ĺnidade. perfazendo um total de 65 casos descohertos no ano.

Assim. a incidência esperada era 140 e a observada de 37. ou seja. a eficácia da descoberta de casos seria apenas $26.4 \%$ e. conseqüentemente. 103 casos não teriam sido detectados.

Considerando todos os casos. inclusive os positivos apenas à cultura $(35 \%)$. a incidencia esperada seria 215 . dos quais apenas 65 (30's) diagnosticados. Se essas estimativas de noros infectados (1.816) e doentes (215) estiverem corretas. cada doente infecta. em média. 8 pessoas por ano nessa área.

Os fatores que poderiam explicar essa haixa eficácia seriam:

1. o método usado para calcular o risco de infecção seria inadequado. não acreditamos que esse fator pudesse ser o responsável uma vez que aplicamos metodologia já estudada e testada. ficando assim essa hipótese afastada;

2. a relação da incidência igual 60 vezes o valor do risco de infecção poderia não ser verdadeira para a área em pauta. onde são executadas atividades antituberculose; aliás, os próprios autores ressalvam que a relação seria aplicável somente em comunidades onde não existem programas efetivos de controle da doença;

3. ressalvadas essas possiveis causas de erro. ainda assim a eficácia estimada revelou-se muito abaixo do esperado, sulgerindo que não se trata apenas de deficiências relacionadas com a metodologia usada. O que não é surpresa. considerando-se que se trata de Lnidade Sanitária estática com minima penetração junto à comunidade onde atua.

A dinamização das atividades. alicerçadas em programa educativo especialmente dirigido aos residentes em bairros economicamente menos favorecidos. poderá contrihuir para esclarecer as dúvidas aventadas.

\section{CONCLUSOES}

1. O risco anual de infecção tuherculosa na população geral é de $1.02 \%$.

2. A incidência anual de casos bacilíferos lao exame bacterioscópico e/ou cultura) deve ser superior à obserrada.

3. Se as estimativas de incidencia de infectados e de casos novos estiverem corretas. cada fonte bacilifera infecta nessa área, em média, 8 pessoas por ano. 
RUFFINO NETTO, A. \& ARANTES, G. R. - Risco de infeção tuberculosa em município do interior do Estado de São Paulo e suas aplicações. Rev. Saúde públ., S. Paulo, $10: 143-9,1976$.

RSPU-B/308

Ruffino Netto, A. \& ARAntes, G. G. - [Risk of tuberculous infection in a community in the State of S. Paulo (Brazil) and its applications]. Rev. Saúde públ., S. Paulo, 10:143-9, 1976.

SUMMARY: The risk estimation of tuberculosis infection in the population of Ribeirão Preto, State of S. Paulo (Brazil), based on a transversal study, as well as on the annual incidence of positive sputum smear cases in that community is proposed. Data from 35.680 persons living in that community which had been registered at the local Public Health Center were used for this estimation. The risk of infection for different age groups and for the whole population as well its variations and implications was analysed.

UNITERms: Tuberculosis infection, risk. Prevalence and incidence. S. Paulo State (Brazil).

\section{REFERENCIAS BIBLIOGRÁFICAS}

1. ARANTES, G. R. - Avalıação de serviço anti-tuberculose na rotina de Saúde Pública. Rev. Saúde publ., S. Paulo, 8:105-18, 1974 .

2. ARANTES, $G$ R. et al. - Interpretaçāo da sensibilidade tuberculinica em população do interior do Estado de São Paulo. [Apresentado no XVII Congresso Nacional de Tuberculose e Doenças Respiratórias. Brasilia. DF, $1975]$.

3. NARAIN, R. et al. - Problems connected with estimating the incidence of tuberculosis infection. Bull. Wld. Hlth. Org., 34:605-22. 1966.

4. RLFFINO NETTO, A. - Epudemiologia da tuberculose: estudos de alguns asper- tos ligados a modelos de prevençán, diagnóstico e modelos epidemiométricos. Ribeirão Preto, SP, 1975 [Tese de 1ivre-docência - Faculdade de Medicina de Ribeirão Preto da USP].

5. RLFFINo NetTo. A et al. - Alergia tuberculinica pós-vacinação com B.C.G. intradérmico e pós-infeçãa natural. [Apresentado no XVII Congresso Nacional de Tuberculose e Doenças Respiratórias. Brasilia. DF, 1975].

6. STYBLO. K. \& StTHERLAND, I. - Epidemiological Indices for planning, surverllance and evaluation of tuberculosis programms Bull. int. Un. Tuberc., 49:66-73, 1974 .

Recebido para publicașo em 03/12/19r5 Aprovado para publicactio em 05/01/19r6 